\title{
Polychromatic digital holographic microscopy: a quasicoherent-noise-free imaging technique to explore the connectivity of living neuronal networks (Publisher's Note)
}

Céline Larivière-Loiselle, ${ }^{\text {a,b }}$ Erik Bélanger, ${ }^{\text {a,b,c }}$ and Pierre Marquet ${ }^{\text {a,c,d }}$

${ }^{a}$ Université Laval, Centre de recherche CERVO, Québec, Canada

${ }^{\text {b} U n i v e r s i t e ́ ~ L a v a l, ~ D e ́ p a r t e m e n t ~ d e ~ p h y s i q u e, ~ d e ~ g e ́ n i e ~ p h y s i q u e ~ e t ~ d ' o p t i q u e, ~}$

Faculté des sciences et de génie, Québec, Canada

${ }^{c}$ Université Laval, Centre d'optique, photonique et laser, Québec, Canada

${ }^{\mathrm{d} U n i v e r s i t e ́ ~ L a v a l, ~ D e ́ p a r t e m e n t ~ d e ~ p s y c h i a t r i e ~ e t ~ n e u r o s c i e n c e s, ~ F a c u l t e ́ ~ d e ~ m e ́ d e c i n e, ~}$

Québec, Canada

[DOI: 10.1117/1.NPh.8.1.019801]

This article [Neurophotonics 7(4), 040501 (2020) doi: 10.1117/1.NPh.7.4.040501] was originally published on 16 October 2020 with a subsection missing from Sec. 3, Results and Discussion: "Disperson of the Observed Cells," Sec. 3.3, containing a single paragraph, was inadvertently excised from the galley proof in the course of correction, causing loss of information for the reader.

The missing section heading and paragraph were restored on 20 January 2021. As part of that correction, a reference citation that had been erroneously repositioned in Sec. 2.2 (Ref. 33) was restored to Sec. 3.3. 